\title{
Graphene saturable absorbers for VECSELs
}

\author{
V. J. Wittwer*a, C. A Zaugg ${ }^{\mathrm{b}}$, Z. Sun ${ }^{\mathrm{a}}$, D. Popa ${ }^{\mathrm{a}}$, S. Milana ${ }^{\mathrm{a}}$, T. S. Kulmala ${ }^{\mathrm{a}}$, R. S. Sundaram ${ }^{\mathrm{a}}$, \\ M. Mangold ${ }^{\mathrm{b}}$, M. Golling ${ }^{\mathrm{b}}$, Y. Lee ${ }^{\mathrm{c}}$, J. H. Ahn $^{\mathrm{c}}, \mathrm{U}$. Keller ${ }^{\mathrm{b}}$, and A. C. Ferrari ${ }^{\mathrm{a}}$ \\ ${ }^{a}$ Cambridge Graphene Centre, University of Cambridge, Cambridge CB3 0FA, UK; \\ ${ }^{b}$ Department of Physics, Institute for Quantum Electronics, ETH Zürich, 8093 Zürich, Switzerland; \\ ${ }^{\text {c} S c h o o l ~ o f ~ E l e c t r i c a l ~ \& ~ E l e c t r o n i c ~ E n g i n e e r i n g, ~ Y o n s e i ~ U n i v e r s i t y, ~ S e o u l ~ 120-749 ~ a n d ~ S K K U ~}$ \\ Advanced Institute of Nanotechnology, Sungkyunkwan University, Suwon, 440-746, Korea
}

\begin{abstract}
We report mode-locking of an optically pumped VECSEL using a graphene-based saturable absorber mirror (GSAM). Self-starting and stable modelocked operation is demonstrated with $473 \mathrm{fs}$ pulses at $1.5 \mathrm{GHz}$ repetition rate and $949 \mathrm{~nm}$ center wavelength. Wavelength tuning is achieved over a $46 \mathrm{~nm}$ bandwidth. We discuss the mirror design, the fabrication of the GSAMs, and give an outlook on further optimization of the design, including dielectric top coatings to protect the graphene and to increase the flexibility in the design.
\end{abstract}

Keywords: VECSEL, graphene, mode-locking, broadband saturable absorber

\section{INTRODUCTION}

Ultrafast vertical-external-cavity surface-emitting lasers (VECSELs), ${ }^{1,2}$ are excellent pulsed sources for various applications, such as multi-photon microscopy, ${ }^{3}$ optical data communications, ${ }^{2}$ supercontinuum generation ${ }^{4}$ and ultracompact stabilized frequency combs. ${ }^{1}$ VECSELs combine the advantages of semiconductor lasers, such as compactness, ${ }^{5}$ with those of diode pumped solid-state lasers, such as low timing jitter ${ }^{6}$, excellent beam quality, ${ }^{7}$ high average ${ }^{7,8}$ and peak power ${ }^{4,} 9$. The conventional devices used for modelocking of VECSELs are semiconductor saturable absorber mirrors (SESAMs) ${ }^{10}$ since they offer advantages such as an excellent ratio of saturable to non-saturable losses (e.g. $\left.50: 1^{11}\right)$ and a high damage threshold $\left(>0.21 \mathrm{~J} / \mathrm{cm}^{2}\right)^{11}$. However, SESAMs, epitaxially grown on lattice-matched semiconductor substrates ${ }^{10}$, only offer a limited operation bandwidth (to date, the broadest tuning range of VECSELs mode-locked with SESAMs is $13.7 \mathrm{~nm})^{12}$ and have a recovery time ranging from several hundreds of $\mathrm{fs}^{13}$ to tens of $\mathrm{ps}^{11}$ Graphene based saturable absorbers (GSAs) have emerged as a promising SAs for ultrafast pulse generation, since graphene offers broadband operation and ultrafast recombination dynamics in the order of tens of fs. ${ }^{14}$, 15 High quality, single layer graphene (SLG) can be grown ${ }^{16}$ and integrated in a variety of lasers. ${ }^{17}$ In common linear cavities, SLG is used as SA in transmission ${ }^{18,19}$ resulting in a double-pass per round-trip. The unsaturated loss in such a configuration is typically $\sim 2 \cdot 2.3 \%$ (the factor 2 accounts for the double-pass). While this allows to use GSA to modelock fiber ${ }^{16,20,21}$, solid-state $^{17}, 19$ and waveguide ${ }^{22}$ lasers, it poses limitations for VECSELs ${ }^{1}$. These typically require a SA with losses $<3 \%$ per cavity round-trip. ${ }^{23}{ }^{24}$ Therefore, inserting a GSA in transmission (e.g. SLG on a quartz substrate ${ }^{19}$ ) inhibits lasing. To achieve self-starting passive modelocking of a VECSEL using a GSA it is crucial to reduce the total cavity roundtrip losses to $<3 \%$ while maintaining a modulation depth in the range $0.5-2 \%$. ${ }^{2}$ We achieved this in Ref. 24 by controlling the electric field intensity in SLG on a high-reflection mirror. Here we give an outlook on the optimization of the design of the saturable absorber mirror, such as the use of a dielectric top-coating.

*vw263@cam.ac.uk, Telephone: +44 (0)1223 748347, www.graphene.cam.ac.uk 


\section{MIRROR DESIGN}

The structure consists of a highly reflecting mirror with a spacer and SLG on top. The thickness of the spacer changes the field intensity enhancement at the SLG and thus determines the absorption of the device. ${ }^{24}$ The field intensity enhancement for a design wavelength $\lambda$ in function of the thickness of the spacer layer $d$ is then ${ }^{24}$ :

$$
\xi_{\text {abs }}(d) \approx \frac{4}{1+n^{2} \cot ^{2}\left(\frac{2 \pi}{\lambda} n d\right)},
$$

where $n$ is the refractive index of the spacer layer material. Using $\mathrm{SiO}_{2}$ as spacer with thickness $0, \lambda / 12 \mathrm{SiO}_{2}, \lambda / 8 \mathrm{SiO}_{2}$ and $\lambda / 4 \mathrm{SiO}_{2}$, the field intensity enhancement at the location of SLG $\xi_{\text {abs }}$ is $0,0.5,1.3$ and 4 (Figure 1 and Eq. 1). The absorption is ${ }^{24} A=\xi_{\text {abs }} \cdot 2.3 \%$, where $2.3 \%$ is the absorption for SLG in transmission (corresponding to $\xi_{\text {abs }}=1$ ).

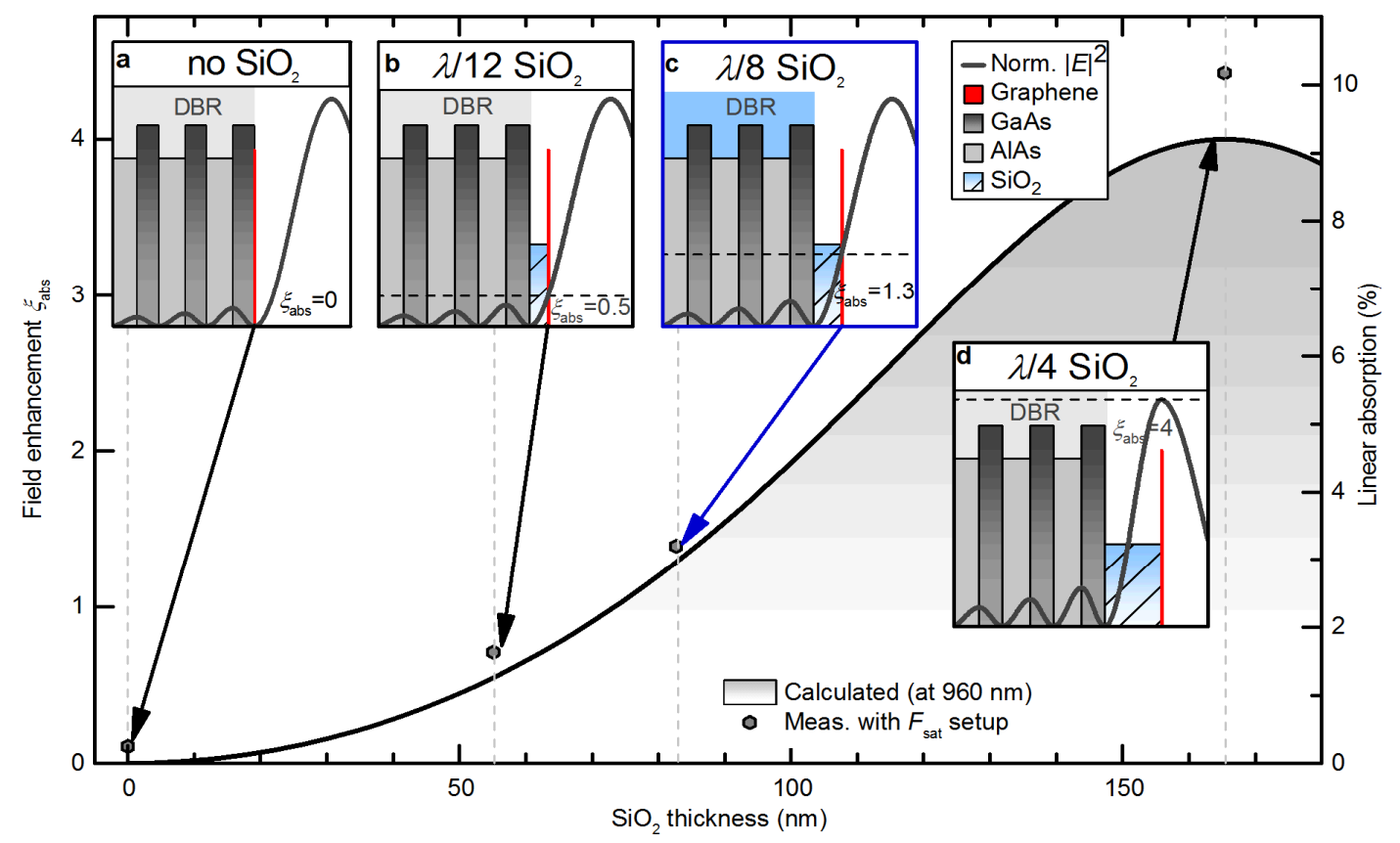

Figure 1. Spacer layer thickness dependent field intensity enhancement at the SLG location $\xi_{\text {abs }}$ (left axis) and corresponding linear absorption (right axis), calculated (black line) compared to the absorption measurements (dots). Insets: Schematic view of four structures showing the last DBR mirror pairs with a) no $\mathrm{SiO}_{2}$, b) $\lambda / 12 \mathrm{SiO}_{2}(55 \mathrm{~nm})$, c) $\lambda / 8 \mathrm{SiO}_{2}(83 \mathrm{~nm})$ and d) $\lambda / 4 \mathrm{SiO}_{2}(165 \mathrm{~nm})$. The dark gray curve shows the normalized standing wave electric field intensity (for the design wavelength $\lambda=960 \mathrm{~nm}$ ) as a function of vertical displacement from the mirror surface. SLG (red) is the top layer.

\section{FABRICATION AND CHARACTERIZATION}

Four GSAMs are fabricated with spacers between the mirror and SLG by coating the mirror with: $0, \lambda / 12 \mathrm{SiO}_{2}, \lambda / 8 \mathrm{SiO}_{2}$ and $\lambda / 4 \mathrm{SiO}_{2}$. The high-reflection mirrors are anti-resonant distributed Bragg reflectors (DBRs). ${ }^{25,}{ }^{26}$ DBRs typically consist of a stack of multiple layers with alternating high and low refractive index ${ }^{25,26}$, each with an optical thickness of a quarter of the design wavelength. The partial reflections at the layer interfaces interfere constructively resulting in high reflection $(\sim 100 \%){ }^{25,26}$ We use 30-pair anti-resonant AlAs/GaAs $(81.1 \mathrm{~nm} / 67.85 \mathrm{~nm})$ DBRs, designed to have a node of the standing wave at the surface of the top layer (anti-resonance) and a reflectivity $>99.997 \%$ at $960 \mathrm{~nm}$. The DBRs are grown on a 3 inch $600 \mu \mathrm{m}$ thick GaAs by molecular beam epitaxy (MBE), then cleaved into square $1 \mathrm{~cm}^{2}$ pieces. Subsequently, the $\mathrm{SiO}_{2}$ layers are deposited by plasma enhanced chemical vapor deposition with thickness of $0 \mathrm{~nm}$, $55 \mathrm{~nm}(\lambda / 12), 83 \mathrm{~nm}(\lambda / 8)$ and $165 \mathrm{~nm}(\lambda / 4)$ on different DBR samples (Figure 1(a-d)).

The SLG is grown by chemical vapor deposition (CVD) as discussed in Ref. 24. Subsequently it is transferred onto the $\mathrm{SiO}_{2}$-coated DBRs. ${ }^{24}$ A picture of the $\lambda / 8 \mathrm{SiO}_{2}$ sample is in Figure 2(a). The linear unsaturated absorption of the four 
samples is measured with a high-precision reflectivity setup ${ }^{27}$ giving $A=0.25 \%$ (no $\left.\mathrm{SiO}_{2}\right), 1.6 \%\left(\lambda / 12 \mathrm{SiO}_{2}\right), 3.2 \%(\lambda / 8$ $\left.\mathrm{SiO}_{2}\right)$ and $10 \%\left(\lambda / 4 \mathrm{SiO}_{2}\right)$ at $960 \mathrm{~nm}$. In Figure 1 those measurements show good agreement with the calculated values based on Eq. 1. Figure 2(b) plots the field intensity enhancement calculated from Eq.1 as a function of wavelength. The GSAMs reflectivity as a function of input light fluence $\left(\mathrm{J} / \mathrm{cm}^{2}\right)$ is characterized using the setup described in Ref. 27. The measurement for the $\lambda / 4 \quad \mathrm{SiO}_{2}$ device (the GSAM with $\xi_{\text {abs }}=4$ ) reveals a saturation fluence $\mathrm{F}_{\text {sat }} \sim 100 \mu \mathrm{J} / \mathrm{cm}^{2}$ (corresponding to a peak intensity Ipeak $\sim 1.0 \mathrm{GW} / \mathrm{cm}^{2}$ ) and a modulation depth $\sim 5 \%{ }^{24}$ The $\mathrm{F}_{\text {sat }}$ of the $\lambda / 8$ sample is estimated $\sim 200 \mu \mathrm{J} / \mathrm{cm}^{2}$, higher than the $\lambda / 4$ sample, because the smaller field intensity enhancement at the absorber makes the device saturate at a higher fluence. ${ }^{24}$
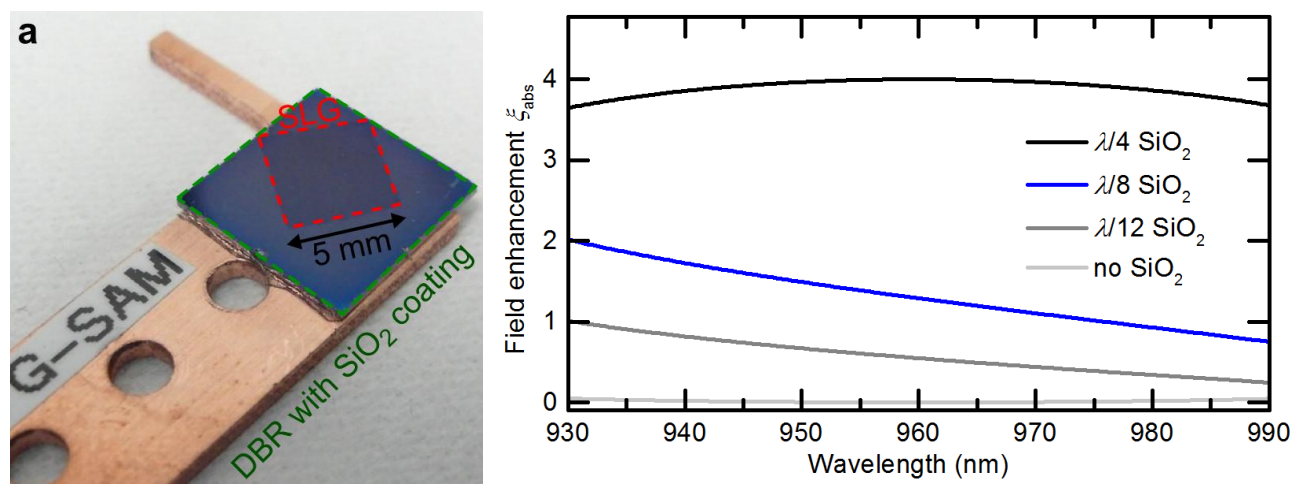

Figure 2. a) $\lambda / 8$ GSAM used for the modelocking experiments: SLG transferred onto the AlAs/GaAs DBR with a layer of $83 \mathrm{~nm} \mathrm{SiO}$. The SLG is seen as shaded area, since the $83 \mathrm{~nm} \mathrm{SiO}$ thickness gives a high optical contrast in the visible range $^{28}$. b) Field intensity enhancement at the absorber vs. wavelength.

\section{MODELOCKING AND WAVELENGTH TUNING}
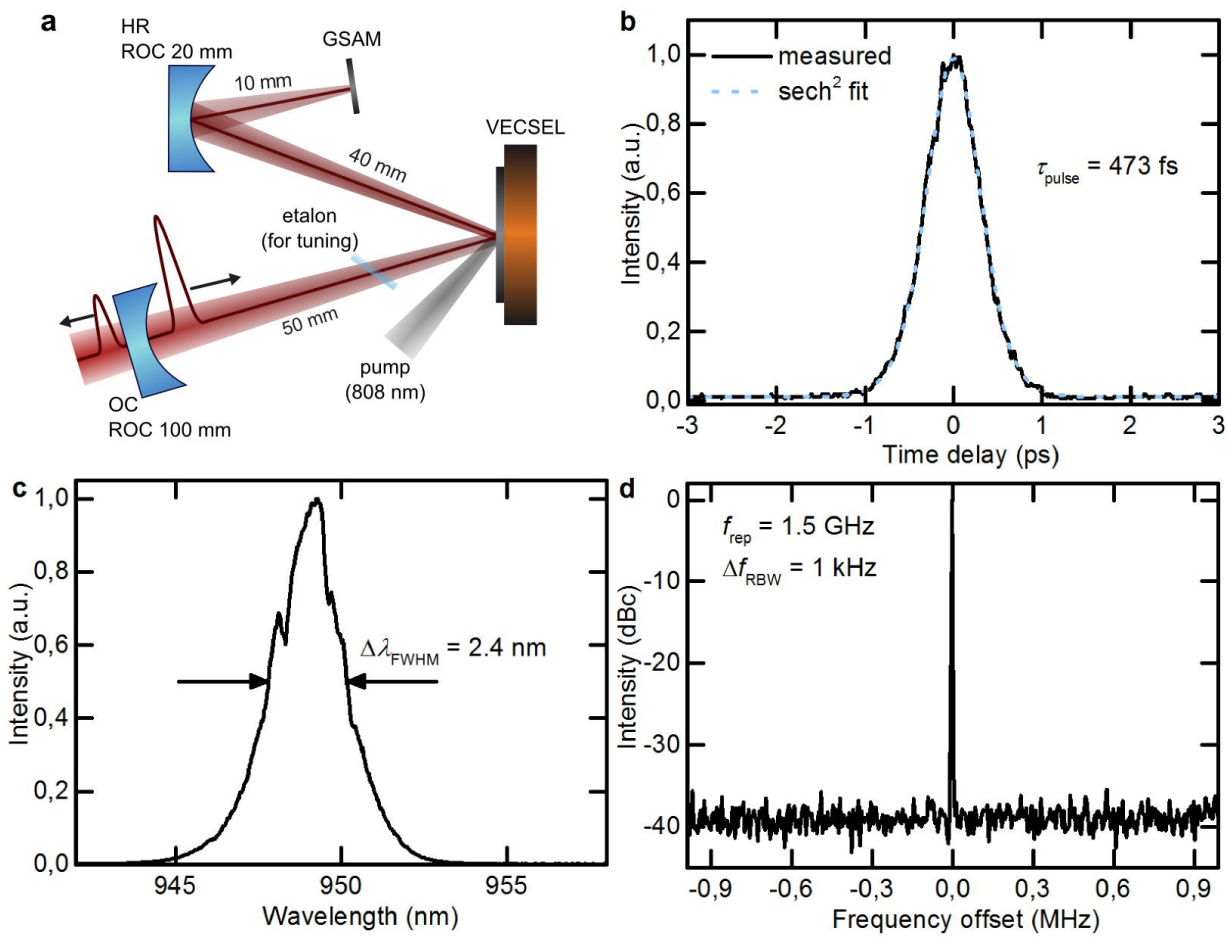

Figure 3. a) Sketch of the laser cavity, b) intensity autocorrelation trace and the fit to the auto correlation of a $473 \mathrm{fs}^{\mathrm{sech}}{ }^{2}$ pulse, c) optical spectrum and d) Microwave spectrum of the repetition rate centered at $1.5 \mathrm{GHz}$ and measured with a resolution bandwidth of $1 \mathrm{kHz}$. 
To demonstrate the suitability for passive modelocking the $\lambda / 8$ GSAM sample is used in a VECSEL cavity. The laser cavity setup is in Figure 3(a). The resonator mode and pump spot radius on the gain chip are 150 and $\sim 30 \mu \mathrm{m}$ on the absorber, using a concave folding mirror with $20 \mathrm{~mm}$ radius of curvature. With a quantum dot (QD) VECSEL gain chip we obtain stable modelocking with pulse duration of $473 \mathrm{fs}$, as shown in the autocorrelation trace in Figure 3(b). The spectrum is centered at $\sim 950 \mathrm{~nm}$ (Figure 3e) and the repetition rate is $1.5 \mathrm{GHz}$ (consistent with the $10 \mathrm{~cm}$ cavity length). The average output power is $5 \mathrm{~mW}$ using an output coupling (OC) mirror with $0.1 \%$ transmission. The pulse fluence on the GSAM is $\sim 125 \mu \mathrm{J} / \mathrm{cm}^{2}$. The broad operation bandwidth is demonstrated using three VECSELs optimized for emission at 940,950 and $970 \mathrm{~nm}$. An antireflection coated $20 \mu \mathrm{m}$ thick fused silica etalon is used for wavelength tuning. The GSAM is not temperature controlled, and supports modelocking in a tuning range $\sim 46 \mathrm{~nm}$, from 935 to $981 \mathrm{~nm}$. ${ }^{24}$

\section{PROSPECTIVE DESIGN OPTIMIZATION}

The approach to optimize the spectral flatness of the device is to place the absorber in the antinode of the standing wave (in the field maximum) for the design wavelength. This is the case for the $\lambda / 4$ GSAM (Figure 1d). But, this has $\xi_{\mathrm{abs}}=4$, therefore too high absorption for VECSEL modelocking. By adding an additional dielectric layer on top of the SLG the field may be reduced, while keeping the SLG still in the anti-node. This is shown in Figure 4(b). Here a $\lambda / 4 \mathrm{SiO}_{2}$ $(165 \mathrm{~nm})$ layer between DBR and graphene places the absorber in the anti-node and a $\lambda / 4 \mathrm{Al}_{2} \mathrm{O}_{3}(136 \mathrm{~nm})$ layer reduces the field. Figure 4(b) shows the design of the $\lambda / 8$ GSAM for comparison, with the same field intensity enhancement at the design wavelength of $960 \mathrm{~nm}$.
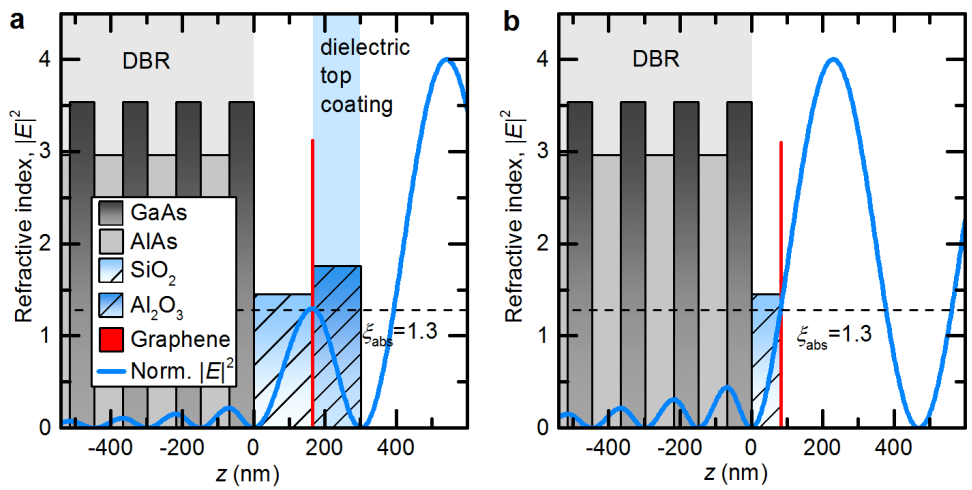

Figure 4. a) Design with additional dielectric layer as top coating. Here the absorber is placed in the antinode and the field is reduced with the top coating to the field intensity enhancement of $\xi_{\mathrm{abs}}=1.3$, b) design used for modelocking and the broadband tuning experiment with field intensity enhancement of 1.3 (at $960 \mathrm{~nm}$ ) at the SLG absorber position.

In Figure 5 the field intensity enhancement is computed (using the transfer matrix algorithm for multilayer structures ${ }^{29}$ ) in the wavelength range between 920 and $1000 \mathrm{~nm}$. This shows an improvement in the wavelength independence of the field intensity enhancement, therefore absorption of the device, in comparison to the $\lambda / 8$ GSAM. Furthermore this coating also acts as a protective top-coating.

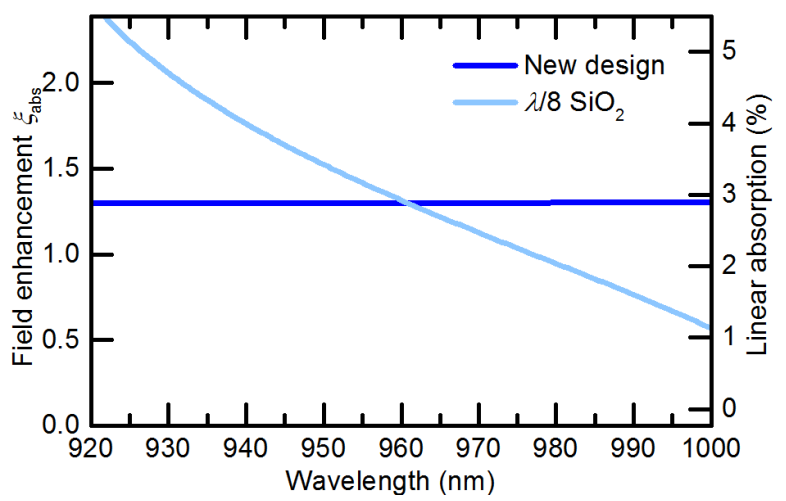

Figure 5. Field intensity enhancement at the absorber vs. wavelength for the new design. The new design (dark blue) has a much weaker wavelength dependence compared to the $\lambda / 8 \mathrm{SiO}_{2}$ sample (light blue). 


\section{CONCLUSIONS}

We reported on an approach to control the absorption of graphene on a mirror in the range between $0-10 \%$. This enabled us to fabricate a GSAM for VECSEL modelocking with sub $500 \mathrm{fs}$ pulses at $1.5 \mathrm{GHz}$ pulse repetition rate. Wavelength tuning was achieved over a $46 \mathrm{~nm}$ bandwidth. We gave an outlook on further optimization of the design, including dielectric top coatings to protect the graphene and to further increase the spectral flatness.

\section{ACKNOWLEDGMENTS}

We thank T. Südmeyer for useful discussions. We acknowledge funding from a Royal Society Wolfson Research Merit Award, the European Research Council Grants NANOPOTS, Hetero2D, EU grants RODIN, GENIUS, MEM4WIN, CareRAMM, and Graphene Flagship (contract no. NECT-ICT-604391), EPSRC grants EP/K01711X/1, EP/K017144/1, Nokia Research Centre, Emmanuel College, Cambridge, the FIRST clean room facility of ETH, the Swiss National Science Foundation (SNSF) and the Swiss Confederation Program Nano-Tera.ch, which was scientifically evaluated by the SNSF.

\section{REFERENCES}

[1] Keller, U., and Tropper, A. C., "Passively modelocked surface-emitting semiconductor lasers," Phys. Rep., 429(2), 67-120 (2006).

[2] Südmeyer, T., Maas, D. J. H. C., and Keller, U., [Mode-Locked Semiconductor Disk Lasers] Wiley-VCH Verlag GmbH \& Co. KGaA, (2010).

[3] Aviles-Espinosa, R., Filippidis, G., Hamilton, C., Malcolm, G., Weingarten, K. J., Südmeyer, T., Barbarin, Y., Keller, U., Santos, S. I. C. O., Artigas, D., and Loza-Alvarez, P., "Compact ultrafast semiconductor disk laser: targeting GFP based nonlinear applications in living organisms," Biomed. Opt. Express, 2(4), 739-747 (2011).

[4] Wilcox, K. G., Tropper, A. C., Beere, H. E., Ritchie, D. A., Kunert, B., Heinen, B., and Stolz, W., "4.35 kW peak power femtosecond pulse mode-locked VECSEL for supercontinuum generation," Opt. Express, 21(2), 1599-1605 (2013).

[5] Lorenser, D., Maas, D. J. H. C., Unold, H. J., A.-R. Bellancourt, Rudin, B., Gini, E., Ebling, D., and Keller, U., "50-GHz passively mode-locked surface-emitting semiconductor laser with $100 \mathrm{~mW}$ average output power," IEEE J. Quantum Electron., 42(8), 838-847 (2006).

[6] Wittwer, V. J., Zaugg, C. A., Pallmann, W. P., Oehler, A. E. H., Rudin, B., Hoffmann, M., Golling, M., Barbarin, Y., Sudmeyer, T., and Keller, U., "Timing Jitter Characterization of a Free-Running SESAM Modelocked VECSEL," Photonics Journal, IEEE, 3(4), 658-664 (2011).

[7] Rudin, B., Wittwer, V. J., Maas, D. J. H. C., Hoffmann, M., Sieber, O. D., Barbarin, Y., Golling, M., Südmeyer, T., and Keller, U., "High-power MIXSEL: an integrated ultrafast semiconductor laser with $6.4 \mathrm{~W}$ average power," Opt. Express, 18(26), 27582-27588 (2010).

[8] Heinen, B., Wang, T. L., Sparenberg, M., Weber, A., Kunert, B., Hader, J., Koch, S. W., Moloney, J. V., Koch, M., and Stolz, W., "106 W continuous-wave output power from vertical-external-cavity surface-emitting laser," Electronics Letters, 48(9), 516-517 (2012).

[9] Scheller, M., Wang, T. L., Kunert, B., Stolz, W., Koch, S. W., and Moloney, J. V., "Passively modelocked VECSEL emitting 682 fs pulses with $5.1 \mathrm{~W}$ of average output power," Electronics Letters, 48(10), 588-589 (2012).

[10]Keller, U., "Recent developments in compact ultrafast lasers," Nature, 424(6950), 831-838 (2003).

[11]Saraceno, C. J., Schriber, C., Mangold, M., Hoffmann, M., Heckl, O. H., Baer, C. R. E., Golling, M., Südmeyer, T., and Keller, U., "SESAMs for high-power oscillators: design guidelines and damage thresholds," IEEE J. Sel. Top. Quantum Electron., 18(1), 29-41 (2012).

[12]Morris, O. J., Wilcox, K. G., Head, C. R., Turnbull, A. P., Mosley, P. J., Quarterman, A. H., Kbashi, H. J., Farrer, I., Beere, H. E., Ritchie, D. A., and Tropper, A. C., "A wavelength tunable 2-ps pulse VECSEL." 824212-824212.

[13]Hoffmann, M., Sieber, O. D., Wittwer, V. J., Krestnikov, I. L., Livshits, D. A., Barbarin, Y., Südmeyer, T., and Keller, U., "Femtosecond high-power quantum dot vertical external cavity surface emitting laser," Optics Express, 19(9), 8108-8116(2011). 
[14]Brida, D., Tomadin, A., Manzoni, C., Kim, Y. J., Lombardo, A., Milana, S., Nair, R. R., Novoselov, K. S., Ferrari, A. C., Cerullo, G., and Polini, M., "Ultrafast collinear scattering and carrier multiplication in graphene," Nat Commun, 4, (2013).

[15]Tomadin, A., Brida, D., Cerullo, G., Ferrari, A. C., and Polini, M., "Nonequilibrium dynamics of photoexcited electrons in graphene: Collinear scattering, Auger processes, and the impact of screening," Physical Review B, 88(3), 035430 (2013).

[16]Bonaccorso, F., Lombardo, A., Hasan, T., Sun, Z., Colombo, L., and Ferrari, A. C., "Production and processing of graphene and 2d crystals," Materials Today, 15(12), 564-589 (2012).

[17]Bonaccorso, F., Sun, Z., Hasan, T., and Ferrari, A. C., "Graphene photonics and optoelectronics," Nat Photon, 4(9), 611-622 (2010).

[18]Baek, I. H., Lee, H. W., Bae, S., Hong, B. H., Ahn, Y. H., Yeom, D.-I., and Rotermund, F., "Efficient ModeLocking of Sub-70-fs Ti:Sapphire Laser by Graphene Saturable Absorber," Applied Physics Express, 5(3), 032701 (2012).

[19]Lagatsky, A. A., Sun, Z., Kulmala, T. S., Sundaram, R. S., Milana, S., Torrisi, F., Antipov, O. L., Lee, Y., Ahn, J. H., Brown, C. T. A., Sibbett, W., and Ferrari, A. C., " $2 \mu \mathrm{m}$ Solid-State Laser Mode-locked By Single-Layer Graphene," Applied Physics Letters, 102, 013113 (2013).

[20]Hasan, T., Sun, Z., Wang, F., Bonaccorso, F., Tan, P. H., Rozhin, A. G., and Ferrari, A. C., "Nanotube-Polymer Composites for Ultrafast Photonics," Advanced Materials, 21(38-39), 3874-3899 (2009).

[21]Sun, Z., Hasan, T., Torrisi, F., Popa, D., Privitera, G., Wang, F., Bonaccorso, F., Basko, D. M., and Ferrari, A. C., "Graphene Mode-Locked Ultrafast Laser," ACS Nano, 4(2), 803-810 (2010).

[22]Mary, R., Brown, G., Beecher, S. J., Torrisi, F., Milana, S., Popa, D., Hasan, T., Sun, Z., Lidorikis, E., Ohara, S., Ferrari, A. C., and Kar, A. K., "1.5 GHz picosecond pulse generation from a monolithic waveguide laser with a graphene-film saturable output coupler," Optics Express, 21(7), 7943-7950 (2013).

[23]Mangold, M., Wittwer, V. J., Sieber, O. D., Hoffmann, M., Krestnikov, I. L., Livshits, D. A., Golling, M., Südmeyer, T., and Keller, U., "VECSEL gain characterization," Optics Express, 20(4), 4136-4148 (2012).

[24]Zaugg, C. A., Sun, Z., Wittwer, V. J., Popa, D., Milana, S., Kulmala, T. S., Sundaram, R. S., Mangold, M., Sieber, O. D., Golling, M., Lee, Y., Ahn, J. H., Ferrari, A. C., and Keller, U., "Ultrafast and widely tuneable vertical-external-cavity surface-emitting laser, mode-locked by a graphene-integrated distributed Bragg reflector," Optics Express, 21(25), 31548-31559 (2013).

[25]Spühler, G. J., Weingarten, K. J., Grange, R., Krainer, L., Haiml, M., Liverini, V., Golling, M., Schon, S., and Keller, U., "Semiconductor saturable absorber mirror structures with low saturation fluence," Appl. Phys. B, 81(1), 27-32 (2005).

[26]Renk, K. F., [Basics of laser physics] Springer, (2012).

[27]Maas, D. J. H. C., Rudin, B., Bellancourt, A.-R., Iwaniuk, D., Marchese, S. V., Südmeyer, T., and Keller, U., "High precision optical characterization of semiconductor saturable absorber mirrors," Opt. Express, 16(10), 7571-7579 (2008).

[28]Casiraghi, C., Hartschuh, A., Lidorikis, E., Qian, H., Harutyunyan, H., Gokus, T., Novoselov, K. S., and Ferrari, A. C., "Rayleigh Imaging of Graphene and Graphene Layers," Nano Letters, 7(9), 2711-2717 (2007).

[29]Chilwell, J., and Hodgkinson, I., "Thin-films field-transfer matrix theory of planar multilayer waveguides and reflection from prism-loaded waveguides,” J. Opt. Soc. Am. A, 1(7), 742-753 (1984). 